Case Report

\title{
Isolated intramuscular histoplasmosis in an elderly - diagnosis by fine needle aspiration cytology
}

\author{
Handa U. ${ }^{1}$, Aggarwal P. ${ }^{2}$, Chander J. ${ }^{3}$, Garg S.K. ${ }^{4}$ \\ ${ }^{1}$ Dr. Uma Handa, Professor \& Head, Department of Pathology, ${ }^{2}$ Dr. Phiza Aggarwal, Assistant Professor, Department of \\ Pathology, ${ }^{3}$ Dr. Jagdish Chander, Professor \& Head, Department of Microbiology, ${ }^{4}$ Dr. Sudhir Kumar Garg, Professor \& \\ Head, Department of Orthopaedics, all authors are affiliated with Govt. Medical College \& Hospital, Chandigarh, India.
}

Corresponding Author: Dr. Phiza Aggarwal, Assistant Professor, Departmentof Pathology, Govt. Medical College \& Hospital, Sector-32, Chandigarh, India. Email: drphiza@hotmail.com

\begin{abstract}
Background: Histoplasmosis is a mycotic infection commonly affecting patients with impaired immunity. It mainly manifests as pulmonary or disseminated infection. The diagnosis is often missed in non-endemic regions due to lack of suspicion especially when it involves unusual sites in healthy individuals.

Case: An elderly male presented with solitary left shoulder swelling for the last 2 months. Clinical and radiological findings were suggestive of malignant etiology. Fine needle aspiration cytology from the lesion demonstrated yeast like fungi within the histiocytes. The cytomorphological features were suggestive of Histoplasma which was later confirmed on culture. Patients had an uneventful recovery with anti-fungal treatment.
\end{abstract}

Conclusion: Histoplasma can present in elderly with indolent involvement of uncommon sites. High index of suspicion accompanied by active search using special stains is the key to diagnosis

Key words: histoplasmosis, shoulder, elderly, FNAC

\section{Introduction}

Histoplasmosis is an air-borne mycotic infection caused by a soil based dimorphic fungus Histoplasma capsulatum. It usually causes symptomatic infection in immunocompromised patients where it manifests as pulmonary or disseminated disease.

Rarely, it can involve other isolated organs like intestine [1], oral cavity [2] and musculoskeletal system [3] in patients with impaired immunity. The diagnosis involves demonstration of the fungus on pathologic examination and/or culture of the infected tissue/sample. The present case describes an elderly male presenting with an isolated shoulder mass that was diagnosed as histoplasmosis on aspiration cytology. This type of presentation in a healthy patient is extremely rare and highlights the need for maintaining high index of suspicion even in non-endemic regions.

The present case also discusses the role of aspiration cytology in the diagnosis of this uncommon fungus and its relative advantages and pitfalls in comparison to culture.

\section{Case Report}

An 82-year-old male patient came to orthopaedics outpatient department (OPD) with complaints of progressive swelling over the left shoulder for last 2 months. (Figure 1) The swelling was accompanied by mild pain and moderate restriction in abduction movement at the shoulder joint. There was no history of preceding trauma. He didn't complain of any respiratory, abdominal or genitourinary symptoms. There was no history of fever or significant weight loss. On clinical examination, there was $6 \times 4 \mathrm{~cm}$ firm swelling present over the tip of shoulder and extending anteriorly. There was no local rise of temperature, erythema or tenderness. Laboratory parameters showed mild leucocytosis (12000/cmm). Blood glucose, liver and kidney function tests were in normal range. He was non-reactive for antibodies to human 


\section{Case Report}

immunodeficiency virus (HIV). The clinical features and the preliminary investigations were suggestive of either malignancy or chronic inflammation. Magnetic resonance imaging (MRI) of the shoulder revealed a large well circumscribed intramuscular lesion measuring $62 \times 28 \times 26 \mathrm{~mm}$ in size involving clavicular part of deltoid muscle with peripheral nodular soft tissue component and central necrotic areas. (Figure 2). Complete full thickness tear of supraspinatous and infraspinatous tendons along with mild cortical bone irregularity was also noted. The radiological findings were suggestive of malignant etiology.

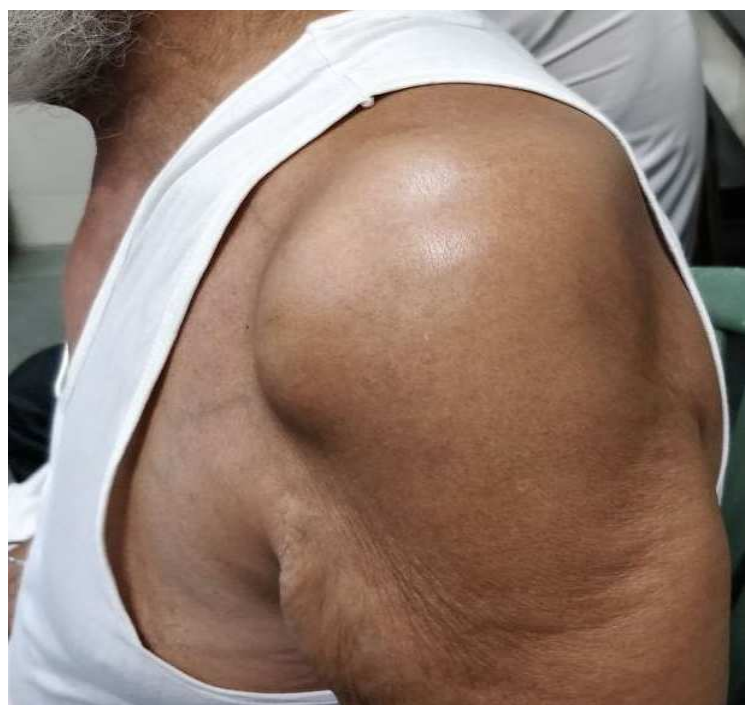

Figure-1: Left shoulder swelling with normal overlying skin

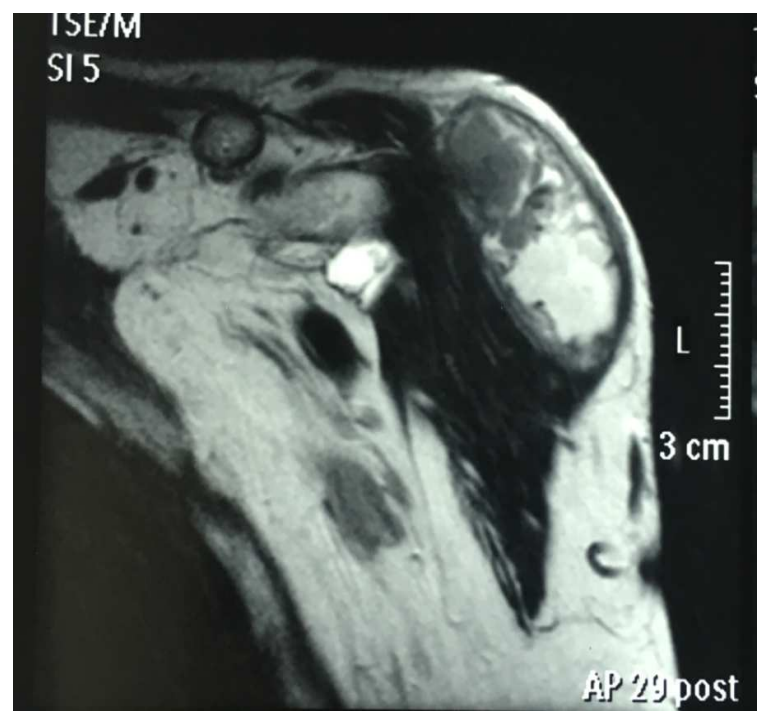

Figure-2: MRI left shoulder (Coronal section) showing a well circumscribed intramuscular lesion involving deltoidmuscle

Patient underwent fine needle aspiration cytology (FNAC) which yielded pus like material. May Grunwald Giemsa (MGG) stained smears showed dense neutrophilic infiltrate along with sheets of histiocytes with formation of ill-defined granulomas. Numerous intracellular (within histiocytes) and extracellular uniform round to oval yeast forms about 2-4 $\mu \mathrm{m}$ in size with a surrounding clear zone were also seen. Few of the yeast forms showed evidence of budding (Figure 3).

Special stains like Gomori methenamine silver (GMS) and Periodic acid-Schiff (PAS) highlighted the fungus. The cytomorphological features were suggestive of Histoplasma species. Subsequently, fungal culture was done which showed the growth of Histoplasma Capsulatum after 4 weeks of incubation. The patient was medically managed with antifungal drugs with which he showed significant improvement.

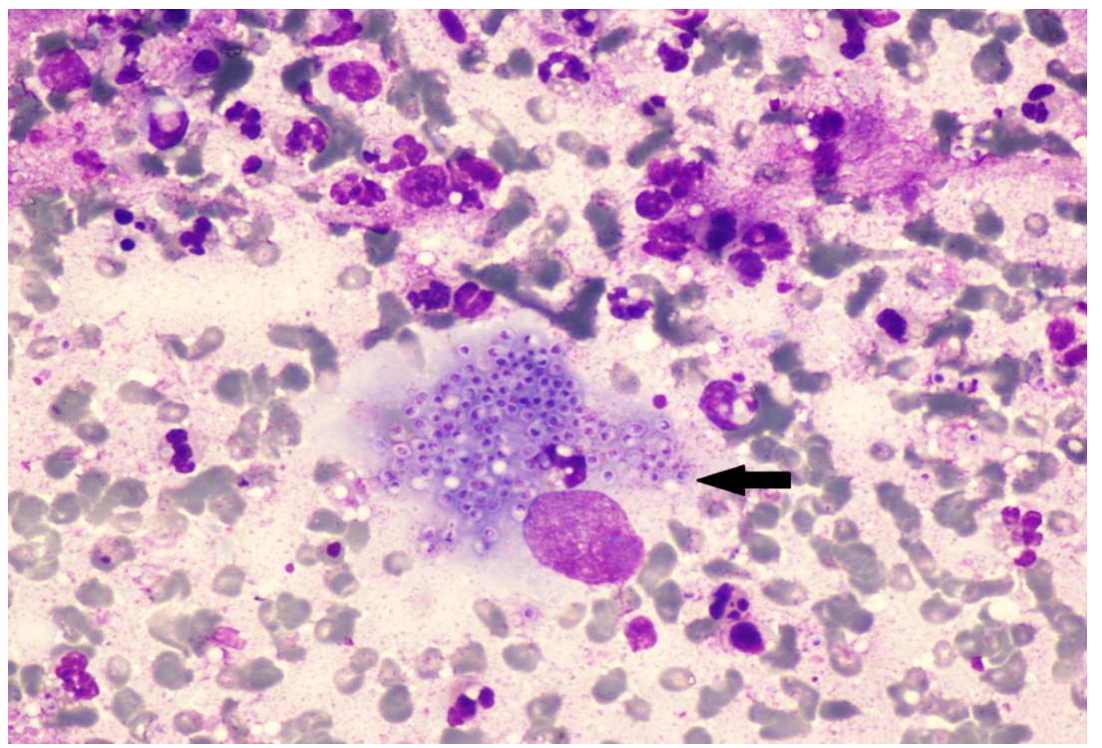

Figure-3: Smear showing numerous intracellular yeasts of

Histoplasma within histiocyte (May Grunwald Giemsa X 400) 


\section{Case Report}

\section{Discussion}

Histoplasmosis is an opportunistic mycosis endemic to certain regions of America and Asia. Considered a rare infection, it is more likely an under recognized entity in India [4]. Different host factors like human immunodeficiency virus, acquired immune deficiency syndrome (HIV/AIDS), solid-organ/bone marrow recipients and diabetes that affect cell mediated immunity increasethe propensity for the disease [5]. The infection usually remains asymptomatic in immunocompetent patients.

However, rarely, it may manifest as localized or disseminated infection in healthy patients causing diagnostic dilemma to the treating physician [6]. There have been few case reports of isolated muscle involvement by Histoplasma in immunocompromised patients [3,7] but its presentation as a solitary shoulder mass in a healthy host, as seen in the patient, has probably not been reported earlier.

There was no evident immunocompromised risk factor in the patient except for extremes of age that might have contributed to increased disease predilection. Likewise, histoplasmosis has been known to occur in elderly but immunocompetent patients previously [8]. The association incites further research is to find out factors that predispose elderly patients to develop fungal infection.

Demonstrating organisms on histopathological examination and/or culture is the gold standard for the diagnosis of histoplasmosis [5]. Presence of caseating or noncaseating granulomas along with demonstration of the fungus using special stains like GMS and PAS help to reach at the diagnosis [9]. Culture remains the gold standard investigation for confirmation of histoplasmosis as well as species typing. However a long lag period of 4-6 weeks and variable sensitivity questions its superiority over pathological isolation $[5,9]$.

Fine needle aspiration cytology (FNAC) is another safe diagnostic procedure routinely used for pathological evaluation of solid and semi-cystic lesions. Its role in the diagnosis of fungal infections has been validated in previous reports [7, 10]. Demonstration of fungus on cytology aspirates from otherwise sterile sites and its cytomorphological analysis to differentiate differentfungi helps to confirm the diagnosis with reasonable accuracy [9]. Certain cytomorphological features based on size, budding (broad based or narrow based) and presence of absence of polysaccharide capsule help to differentiate Histoplasma from other yeasts like fungi i.e. Cryptococcus, Candida glabrata and Blastomyces species on direct microscopy of cytological aspirate. The diagnosis of Histoplasmosis in the present case was also made using the same criteria. The rapidity of reaching the diagnosis makes cytology an initial test of choice that can decrease the time to initiation of treatment.

\section{Conclusion}

The present case highlights a rare presentation of histoplasmosis that mimicked malignancy. High index of suspicion both at the clinician and pathologist end is prerequisite for timely detection of fungal diseases. The report suggests that fungal evaluation using special stains should be included in the routine analysis of cytology samples, especially in elderly patients after excluding malignant etiology. Cytological evaluation has a critical and unchallenging role that can give a prompt diagnosis of histoplasmosis leading to timely intervention.

Funding: Nil, Conflict of interest: None initiated Permission from IRB: Yes

\section{References}

1. Ai XB, Wang ZJ, Dong QC, Lin X, Chen YP, Gong FY, et al. Ileum Histoplasmosis Mimicking Intestinal Tuberculosis and Crohn's Disease. Case Rep Gastroenterol 2018; 12(1): 63-8.doi: 10.1159/000480377

2. Chroboczek T, Dufour J, Renaux A, et al. Histoplasmosis: An oral malignancy-like clinical picture. DOI:10.1016/j. mmcr.2017.11.001

3. Weinberg JM, Ali R, Badve S, Pelker RR. Musculoskeletal histoplasmosis. A case report and review of the literature. J Bone Joint Surg Am. 2001 Nov; 83-A (11): $1718-22$.

4. Gopalakrishnan R, Nambi PS, Ramasubramanian $\mathrm{V}$, et al. Histoplasmosis in India: truly uncommon or uncommonly recognised ? J Assoc Physicians India. 2012 Oct;60:25-8.

5. Azar MM, Hage CA. Clinical Perspectives in the Diagnosis and Management of Histoplasmosis. DOI: 10.1016/j.ccm.2017.04.004

6. Bauddha NK, Jadon RS, Mondal S, Vikram NK, Sood R. Progressive disseminated histoplasmosis in an immunocompetent adult: A case report. Intractable Rare Dis Res 2018; 7(2): 126-9.doi: 10.5582/ irdr. 2018. 01022 . 


\section{Case Report}

7. Goel D, Prayaga AK, Rao N, Damodaram P. Histoplasmosis as a cause of nodular myositis in an AIDS patient diagnosed on fine needle aspiration cytology. A case report. DOI: 10.1159/000325691.

8. Marques SA, Hortense J, Requena CB, et al. Disseminated cutaneous histoplasmosis in elderly patients. An uncommon presentation. DOI:10.1016/j. riam. 2014.02.003.
9. Azar MM, Hage CA. Laboratory Diagnostics for Histoplasmosis. doi: 10. 1128/ JCM. 02430-16. Epub 2017 Mar 8.

10. Gupta N, Arora SK, Rajwanshi A, et al. Histoplasmosis: cytodiagnosis and review of literature with special emphasis on differential diagnosis on cytomorphology. DOI: 10. 1111/j. 1365-2303. 2009. 00693.x

\section{How to cite this article?}

Handa U, Aggarwal P, Chander J, Garg S.K. Isolated intramuscular histoplasmosis in an elderly - diagnosis by fine needle aspiration cytology. Trop J Path Micro 2018; 4(4):349-352.doi:10. 17511/ jopm. 2018.i4.09 